\title{
The CHAOS-3 geomagnetic field model and candidates for the 11th generation IGRF
}

\author{
Nils Olsen ${ }^{1}$, Mioara Mandea ${ }^{2}$, Terence J. Sabaka ${ }^{3}$, and Lars Tøffner-Clausen ${ }^{1}$ \\ ${ }^{1}$ DTU Space, Juliane Maries Vej 30, 2100 Copenhagen, Denmark \\ ${ }^{2}$ Université Paris Diderot, IPG Paris, Géophysique spatiale et planétaire, \\ Bâtiment Lamarck, 5 rue Thomas Mann, 75013 Paris, France \\ ${ }^{3}$ Geodynamics Branch, NASA GSFC, Greenbelt/MD, USA
}

(Received February 2, 2010; Revised June 28, 2010; Accepted July 1, 2010; Online published December 31, 2010)

\begin{abstract}
As a part of the 11th generation IGRF defined by IAGA, we propose a candidate model for the DGRF 2005 , a candidate model for IGRF 2010 and a candidate model for the mean secular variation between 2010 and 2015. These candidate models, the derivation of which is described in the following, are based on the latest model in the CHAOS model series, called "CHAOS-3". This model is derived from more than 10 years of satellite and ground observatory data. Maximum spherical harmonic degree of the static field is $n=60$. The core field time changes are expressed by spherical harmonic expansion coefficients up to $n=20$, described by order 6 splines (with 6-month knot spacing) spanning the time interval 1997.0-2010.0. The third time derivative of the squared magnetic field intensity is regularized at the core-mantle boundary. No spatial regularization is applied.
\end{abstract}

Key words: Geomagnetic reference model, IGRF/DGRF, spherical harmonic analysis.

\section{Introduction}

The 11th generation IGRF is based on seven candidate models for DGRF 2005 and for IGRF 2010, and eight candidate models for the mean secular variation $(\mathrm{SV})$ for 2010-2015. These candidate models have been submitted to IAGA working group V-MOD in October 2009 and evaluated by the IGRF task-force group (see paper by Finlay et al., 2010, this issue). In the following, we describe the derivation of three of the submitted candidate models, one for each of the above mentioned model groups. Our three candidates are based on the CHAOS-3 field model (more specific: CHAOS-3 $\alpha$ ), a new version in the CHAOS model series. Previous versions are CHAOS (Olsen et al., 2006), xCHAOS (Olsen and Mandea, 2008) and CHAOS-2 (Olsen et al., 2009).

Compared to its predecessors, CHAOS-3 is derived from more recent satellite data and using "revised observatory monthly mean values" (which are corrected for external and induced field contributions). In addition, a different regularization scheme is applied to the time changing part of the model.

During the evaluation procedure of the IGRF candidate models it has been found that the degree-1 internal terms of the preliminary model version CHAOS- $3 \alpha$ - derived in September 2009 and used as parent model for our IGRF candidate models-for epoch 2010.0 are rather different from those of the other candidate models. This is most probably due to the fact that only data until summer 2009

Copyright (C) The Society of Geomagnetism and Earth, Planetary and Space Sciences (SGEPSS); The Seismological Society of Japan; The Volcanological Society of Japan; The Geodetic Society of Japan; The Japanese Society for Planetary Sciences; TERRAPUB.

doi:10.5047/eps.2010.07.003 have been used for model version CHAOS-3 $\alpha$. It is therefore interesting to investigate the effect of including more recent data on the model behavior near 2010.0. The resulting final model, CHAOS-3, allows us to make a further assessment of our model candidates (which are based on CHAOS $-3 \alpha$ ) and also of the final adopted IGRF model for epoch 2010.0 .

\section{Data}

For CHAOS-3 we use Ørsted scalar data between March 1999 and December 2009, and vector data between March 1999 and December 2004; CHAMP scalar data between August 2000 and December 2009, and vector data between January 2001 and December 2009; and SAC-C scalar data between January 2001 and December 2004. The same data selection criteria as for the CHAOS-2 model (Olsen et al., 2009) are applied.

To extend the model back in time beyond February 1999 we supplement the satellite data with annual differences of revised observatory monthly means of the North, East and Vertical downward components $(X, Y, Z)$ for the time interval 1997.0-2009.5. However, contrary to CHAOS-2, for which we have used monthly means calculated in the traditional way as the arithmetic mean of all data of a given month (e.g., Chulliat and Telali, 2007), we use revised monthly mean values for CHAOS-3, to minimize the influence of external fields, which contribute to the traditional monthly means due to the way these are calculated. The revised monthly means are calculated by removing from the observatory hourly mean values the sum of:

- a model of the ionospheric (plus induced) field as predicted by the CM4 model (Sabaka et al., 2004), parameterized by the 3-monthly means of $F_{10.7}$ solar flux, 


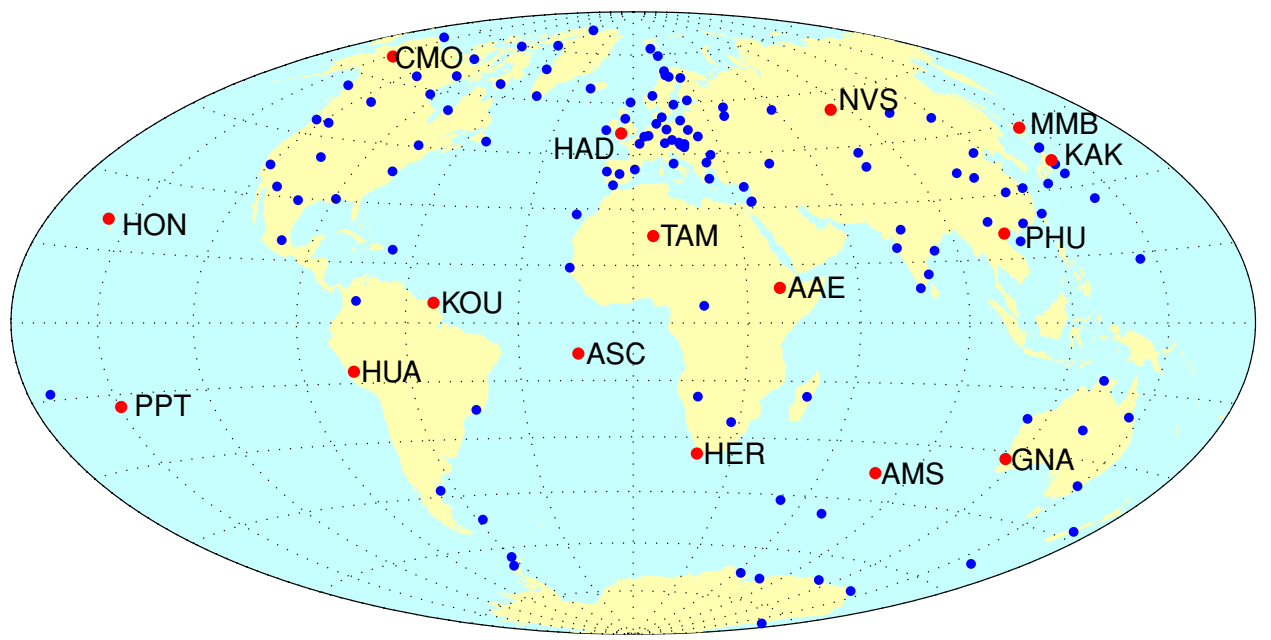

Fig. 1. Location of the 137 observatories used for CHAOS-3 (blue symbols). Emphasized (with red symbols) are the 16 observatories providing data shown in Figs. 5 and 6.

and

- a model of the magnetospheric (plus induced) field as predicted by the external field part of CHAOS-2, parameterized by the $E_{\mathrm{st}}$ and $I_{\mathrm{st}}$ indices.

After subtracting these field corrections from the observed hourly mean values we calculate the robust mean (using Huber weights with tuning constant of 1.5) of all hourly mean values of a given month, each observatory, and each of the three elements $X, Y$ and $Z$. Finally, we take annual differences of the resulting revised monthly means (annual difference value at time $t$ is obtained by taking the difference between those at $t+6$ months and $t-6$ months, thereby eliminating a remaining annual variation in the data). This yields 16,493 values of the first time derivative of the vector components, $d X / d t, d Y / d t, d Z / d t$, for 137 observatories. The location of these observatories are shown in Fig. 1.

A preliminary version of CHAOS-3, named CHAOS-3 $\alpha$, has been used as parent model for our IGRF candidates. These candidates were derived and submitted at the end of September 2009, when the very recent satellite and observatory data were not yet available. Therefore, this preliminary model (CHAOS- $3 \alpha$ ) is based on Ørsted and CHAMP satellite data until August 2009, respectively July 2009, and observatory data until 2009.0. Model parameterization is identical though to that of the final model CHAOS-3, apart from the fact that the extended timespan of the final model leads to slightly more model parameters for describing the timebinned magnetospheric field and the time-varying CHAMP Euler angles, as explained in the following section.

\section{Model Parameterization and Regularization}

The time dependence of core field coefficients up to spherical harmonic degree $n=20$ is described by order 6 B-splines with a 6-month knot separation and five-fold knots at the endpoints, $t=1997.0$ and $t=2010.0$. This yields 27 interior knots (at 1997.5, 1998.0, .., 2009.5) and 6 exterior knots at each endpoint, 1997.0 and 2010.0, resulting in 31 basic B-spline functions, $M_{l}(t)$. Internal field coefficients for degrees $n=21-60$ are static. Time-dependent terms (for degrees $n=1-20$ ) and static terms (for $n=21-$
60) together results in a total of 16,920 internal Gauss coefficients.

Large-scale external (magnetospheric) sources are parameterized in a manner similar to the CHAOS-2 model, with an expansion of the remote magnetospheric sources (magnetotail and magnetopause) in Geocentric Solar Magnetospheric (GSM) coordinates (up to $n=2$ ) and of near magnetospheric sources (magnetospheric ring current) in the Solar Magnetic (SM) coordinate system (also up to $n=2$ ). The time dependence of degree-1 magnetospheric terms in SM coordinates is parameterized by the $E_{\mathrm{st}}$ and $I_{\mathrm{st}}$ indices (Maus and Weidelt, 2004; Olsen et al., 2005). In addition, we solve for large-scale time-varying degree-1 external coefficients in bins of 12 hours length (for $q_{1}^{0}$ ), or 5 days length (for $q_{1}^{1}, s_{1}^{1}$ ), similar as for the CHAOS-2 model. This gives a total of 6,411 external coefficients $(6,151$ for CHAOS-3 $\alpha$, due to the slightly shorter time span).

As part of the field modeling we also perform an inflight instrument calibration and solve for the Euler angles of the rotation between the coordinate systems of the vector magnetometer and of the star sensor providing attitude information. For the Ørsted data, this yields two sets of Euler angles, while for CHAMP we solve for Euler angles in bins of 10 days (i.e. 213 sets of angles). This yields additional $3 \times(2+213)=639$ model parameters $(618$ for CHAOS $-3 \alpha$ ). The total number of model parameters is $16,920+6,411+639=23,970(23,689$ for CHAOS-3 $\alpha)$.

These model parameters are estimated by means of a regularized Iteratively Reweighted Least-Squares approach using Huber weights, minimizing the cost function

$$
\mathbf{e}^{T} \underline{\underline{C}}^{-1} \mathbf{e}+\lambda_{3} \mathbf{m}^{T} \underline{\underline{\Lambda}}_{3} \mathbf{m}+\lambda_{2} \mathbf{m}^{T} \underline{\underline{\Lambda}}_{2} \mathbf{m}
$$

where $\mathbf{m}$ is the model vector, the residuals vector $\mathbf{e}=$ $\mathbf{d}_{\mathrm{obs}}-\mathbf{d}_{\text {mod }}$ is the difference between observation $\mathbf{d}_{\mathrm{obs}}$ and model prediction $\mathbf{d}_{\text {mod }}$, and $\underline{C}$ is the data covariance matrix. Details can be found in Olsen et al. (2009).

$\underline{\Lambda}_{3}$ and $\underline{\underline{\Lambda}}_{2}$ are block diagonal regularization matrices which constrain the third, respectively second, order time derivatives of the core field. $\underline{\underline{\Lambda}}_{3}$ minimizes the mean 
Table 1. Number $N$ of data points, mean, and rms misfit (in nT for the satellite data, and in nT/yr for the observatory data) for CHAOS-3 $\alpha$ and CHAOS-3.

\begin{tabular}{|c|c|c|c|c|c|c|c|c|}
\hline & & & \multicolumn{3}{|c|}{ CHAOS $-3 \alpha$} & \multicolumn{3}{|c|}{ CHAOS-3 } \\
\hline & & component & $N$ & mean & $\mathrm{rms}$ & $N$ & mean & $\mathrm{rms}$ \\
\hline \multirow[t]{12}{*}{ satellite } & \multirow[t]{2}{*}{ all } & $F_{\text {polar }}$ & 298,771 & -0.02 & 5.48 & 314,734 & -0.02 & 5.45 \\
\hline & & $F_{\text {nonpolar }}+B_{B}$ & 824,864 & 0.04 & 2.39 & 855,930 & 0.05 & 2.37 \\
\hline & \multirow[t]{4}{*}{ Ørsted } & $F_{\text {polar }}$ & 114,312 & 0.92 & 4.27 & 120,549 & 0.96 & 4.25 \\
\hline & & $F_{\text {nonpolar }}+B_{B}$ & 412,765 & 0.42 & 2.26 & 425,855 & 0.45 & 2.24 \\
\hline & & $B_{\perp}$ & 144,515 & -0.04 & 7.72 & 144,515 & -0.03 & 7.72 \\
\hline & & $B_{3}$ & 144,515 & -0.01 & 3.62 & 144,515 & -0.01 & 3.62 \\
\hline & \multirow[t]{4}{*}{ CHAMP } & $F_{\text {polar }}$ & 149,130 & -0.86 & 6.63 & 158,856 & -0.89 & 6.57 \\
\hline & & $F_{\text {nonpolar }}+B_{B}$ & 268,559 & -0.59 & 2.47 & 286,535 & -0.61 & 2.46 \\
\hline & & $B_{\perp}$ & 254,289 & 0.01 & 3.50 & 272,123 & 0.01 & 3.51 \\
\hline & & $B_{3}$ & 254,289 & 0.02 & 3.54 & 272,123 & 0.02 & 3.56 \\
\hline & \multirow[t]{2}{*}{ SAC-C } & $F_{\text {polar }}$ & 35,329 & 0.02 & 4.21 & 35,329 & 0.02 & 4.19 \\
\hline & & $F_{\text {nonpolar }}$ & 143,540 & 0.13 & 2.62 & 143,540 & 0.13 & 2.58 \\
\hline \multirow[t]{3}{*}{ observatory } & & $d X / d t$ & 15,756 & -0.26 & 7.26 & 16,493 & -0.28 & 7.15 \\
\hline & & $d Y / d t$ & 15,756 & -0.12 & 4.91 & 16,493 & -0.12 & 4.81 \\
\hline & & $d Z / d t$ & 15,756 & 0.10 & 6.88 & 16,493 & 0.17 & 6.68 \\
\hline
\end{tabular}

squared magnitude of $\left|\frac{\partial^{3} \mathbf{B}}{\partial t^{3}}\right|$, integrated over the core surface $d \Omega$ (radius $c=3485 \mathrm{~km}$ ) and averaged over time:

$$
\left\langle\left|\frac{\partial^{3} \mathbf{B}}{\partial t^{3}}\right|^{2}\right\rangle=\frac{1}{\Delta t} \int_{t=1997}^{2010} \int\left|\frac{\partial^{3} \mathbf{B}}{\partial t^{3}}\right|^{2} d \Omega d t=\mathbf{m}^{T} \underline{\underline{\Lambda}}_{3} \mathbf{m} .
$$

Regularization of the third time derivative alone leads to highly oscillating field behavior. To avoid this we also minimize $|\ddot{\mathbf{B}}|^{2}$ at the core surface at the model endpoints $t=1997.0$ and 2010.0. This is implemented via the regularization matrix $\underline{\Lambda}_{2}$. Note that $\underline{\underline{\Lambda}}$, only acts on 12 (the first and last six) of the 31 spline basis functions. The parameters $\lambda_{3}$ and $\lambda_{2}$ control the strength of the regularization. We considered several values for these two parameters and finally selected $\lambda_{3}=1\left(\mathrm{nT} / \mathrm{yr}^{3}\right)^{-2}$ and $\lambda_{2}=10\left(\mathrm{nT} / \mathrm{yr}^{2}\right)^{-2}$.

This regularization is different from that used for CHAOS-2, for which the time average of the second time derivative, $|\ddot{\mathbf{B}}|^{2}$, is minimized at the core surface. However, we also derive a model using the same model regularization as CHAOS-2s, but applied to the extended data set of CHAOS-3. In the following sections, this model is referred to as the "extended CHAOS-2s" model.

\section{Results and Discussion}

The total number of data points, residual means and root mean squared (rms) values of the two model versions are listed in Table 1. Means and rms are the weighted values calculated from the model residuals $e=d_{\mathrm{obs}}-d_{\text {mod }}$ using the Huber weights $w$ obtained in the last iteration.

The CHAOS-3 rms misfits for the satellite data are slightly lower than those of the CHAOS- $3 \alpha$ model, and lower than those of CHAOS-2 (cf. table 1 of Olsen et al. (2009). Compared to CHAOS-2, most significant is the decrease of the observatory misfit by a factor 2 for the horizontal components $\dot{X}$ and $\dot{Y}$ and by about $30 \%$ for $\dot{Z}$, which is probably due to the use of revised monthly mean values compared to traditional monthly means. In addition to the lower rms misfit, the non-zero means of the observa- tory $\dot{X}$ and $\dot{Z}$ found in CHAOS-2 are no longer present in CHAOS-3, indicating external field contributions in the traditional monthly means that were used for CHAOS-2. The revised monthly means are obviously less contaminated by external field contributions.

Figure 2 shows power spectra of the first time derivative (secular variation, circles) and of the second time derivative (secular acceleration, asterisks). CHAOS-3 has considerably higher secular acceleration power at degrees $n>6$ compared to the two versions of CHAOS-2s, due to the fact that the third time derivative of the field (and not its second time derivative) is regularized. In addition, CHAOS-3 shows a secular acceleration power rather similar in 2005 and 2010. The rather different secular acceleration power of the two versions of CHAOS-2s at epoch 2010 is due to the fact that this epoch is beyond the data span used for CHAOS-2s (only data until March 2009), while data until December 2009 were used for the updated version CHAOS-2s.

Following the approach described in Mandea and Olsen (2006) and Olsen and Mandea (2007) we use CHAMP satellite data to calculate "virtual observatory" monthly mean values from January 2001 to December 2009, from which we derive time series of Gauss coefficients $g_{n}^{m}$ and $h_{n}^{m}$. The CHAMP satellite covers all local times within 132 days, which is roughly 4 months. To minimize the effect of external currents that depend on local time, we have applied a 4-month running mean to the Gauss coefficients. This clearly reduces the scatter of the coefficients. Figures 3 and 4 (updates of figures 3 and 4 from Olsen and Mandea (2007)) show time series of the first time derivative, $d g_{n}^{m} / d t$ and $d h_{n}^{m} / d t$, of the internal Gauss coefficients for $n=1-6$. The symbols present annual differences of the coefficients $g_{n}^{m}, h_{n}^{m}$ while the curves show model values from CHAOS-2s (red), CHAOS- $3 \alpha$ (green) and CHAOS-3 (blue). The magenta curve is for the "extended CHAOS-2s" model. The scatter of the individual monthly solutions (black dots) is largest for the lower-order, and especially for the zonal coefficients, indicating the influence of the 


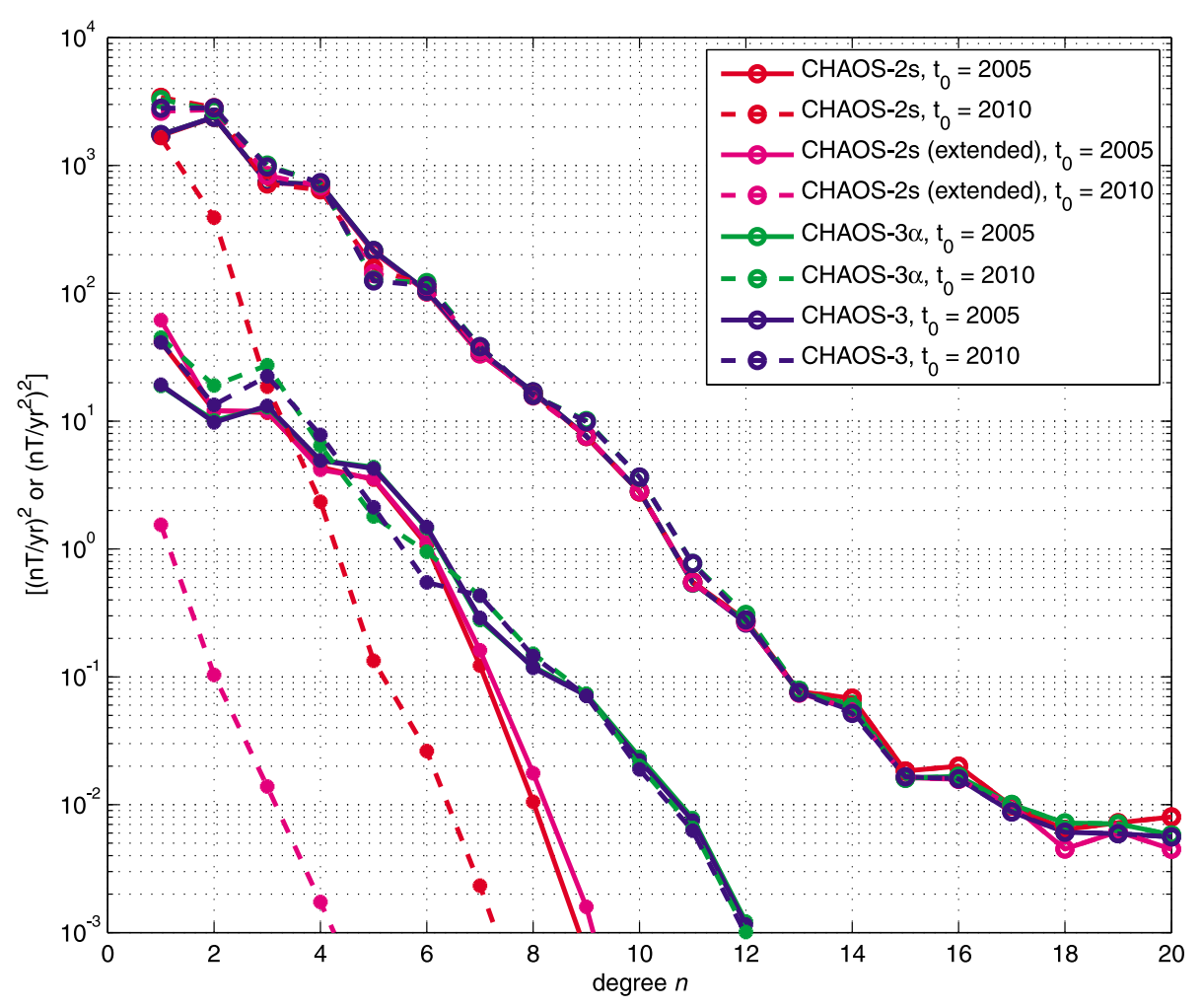

Fig. 2. Power spectra of the first (circles) and second time derivatives (asterisks) for epoch 2005 (solid lines), and 2010 (dashed lines) computed from the four models CHAOS-2s (red), "extended CHAOS-2s" (magenta), CHAOS-3 $\alpha$ (green) and CHAOS-3 (blue).

Table 2. Gauss coefficients $g_{n}^{m}, h_{n}^{m}$ (in nT) and their first time derivative $\dot{g}_{n}^{m}, \dot{h}_{n}^{m}$ (in nT/yr) at epoch 2010.0 for CHAOS-3 $\alpha$, CHAOS-3 and IGRF-11.

\begin{tabular}{r|r|r|r||r|r|r|r|} 
& CHAOS-3 $\alpha$ & CHAOS-3 & IGRF-11 & & CHAOS-3 $\alpha$ & CHAOS-3 & IGRF-11 \\
\hline$g_{1}^{0}$ & -29493.75 & -29496.48 & -29496.5 & $\dot{g}_{1}^{0}$ & 15.34 & 9.43 & 11.4 \\
$g_{1}^{1}$ & -1586.32 & -1586.59 & -1585.9 & $\dot{g}_{1}^{1}$ & 16.10 & 15.54 & 16.7 \\
$h_{1}^{1}$ & 4943.70 & 4944.29 & 4945.1 & $\dot{h}_{1}^{1}$ & -33.79 & -32.54 & -28.8 \\
$g_{2}^{0}$ & -2394.86 & -2396.69 & -2396.6 & $\dot{g}_{2}^{0}$ & -8.94 & -12.20 & -11.3 \\
\hline
\end{tabular}

ionospheric field contributions in the polar ionosphere. The semi-annual variations in some degree-1 coefficients (e.g., $\left.h_{3}^{1}, g_{4}^{1}\right)$ is probably also caused by contributions from polar ionospheric currents. While all four models are in good agreement before 2008, there are considerable differences towards 2010.0. The largest differences are observed for the coefficients $g_{1}^{0}$ and $g_{2}^{0}$, as shown in Table 2, for which the values given by CHAOS- 3 are in a much better agreement to the final IGRF-11, compared with CHAOS-3 $\alpha$.

The time change of the sectorial coefficients $\dot{g}_{n}^{n}, \dot{h}_{n}^{n}$, which represent low-latitude field changes, are better resolved, as indicated by the lower scatter. The temporal variations for the lower degrees are generally well described by the spline models (and especially by CHAOS-3). However, higher degree sectorial terms (e.g., $\dot{h}_{6}^{6}$ ) show rapid field fluctuations that are less well described by the models because of the applied temporal regularization, which increases with degree $n$. Obviously small-scale low-latitude rapid fluctuations exist but are not captured by the present spline models.

A comparison of the annual differences of the observatory monthly means and model estimations are shown in Figs. 5 and 6 for the 16 selected observatories indicated by red symbols in Fig. 1. These observatories are arranged ac- cording to their geographical latitude from North to South. The black symbols are the annual differences of the revised monthly means, used both for CHAOS-3 and CHAOS- $3 \alpha$, while the green symbols represent revised monthly mean values calculated after determination of CHAOS- $3 \alpha$. These values have only been used for estimating CHAOS-3, but not for CHAOS-3 $\alpha$.

For comparison, annual differences of the traditional monthly means (calculated as arithmetic mean of all values of the month) are shown with light blue symbols. Compared to the traditional monthly means these revised monthly means have considerably less scatter; a reduction by a factor of three is not unusual (Olsen, 2009). Their use allows cleaner extraction of the core field signal. It also allows rapid field changes like geomagnetic jerks to be studied, not only in the East-component $Y$ (as is typically done) but also in the other two components $X$ and $Z$ (which are more contaminated by magnetospheric sources and therefore require a careful removal of external fields.) However, despite our attempt to remove ionospheric and magnetospheric fields when calculating revised monthly means there is probably still some contamination from external sources. The peculiar behavior of $\dot{X}$ around 2003 (a year of exception- 

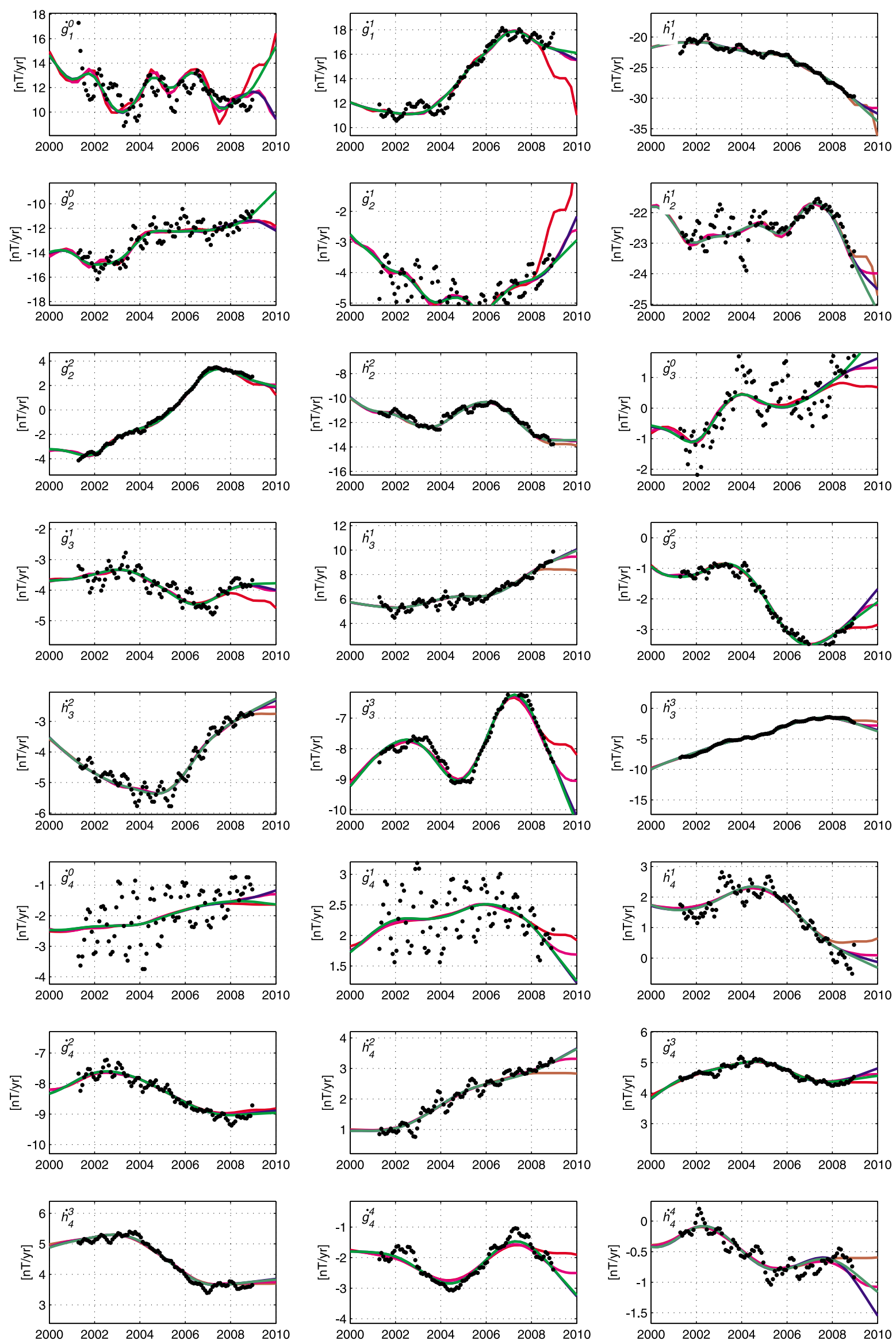

Fig. 3. First time derivatives, $d g_{n}^{m} / d t, d h_{n}^{m} / d t$, of the internal Gauss coefficients, in nT/yr. Symbols represent annual differences of time series of Gauss coefficients obtained from CHAMP "virtual observatories" monthly means, while the curves show model values obtained from CHAOS-2s (red), "extended CHAOS-2s" (magenta), CHAOS-3 $\alpha$ (green) and CHAOS-3 (blue) models. 

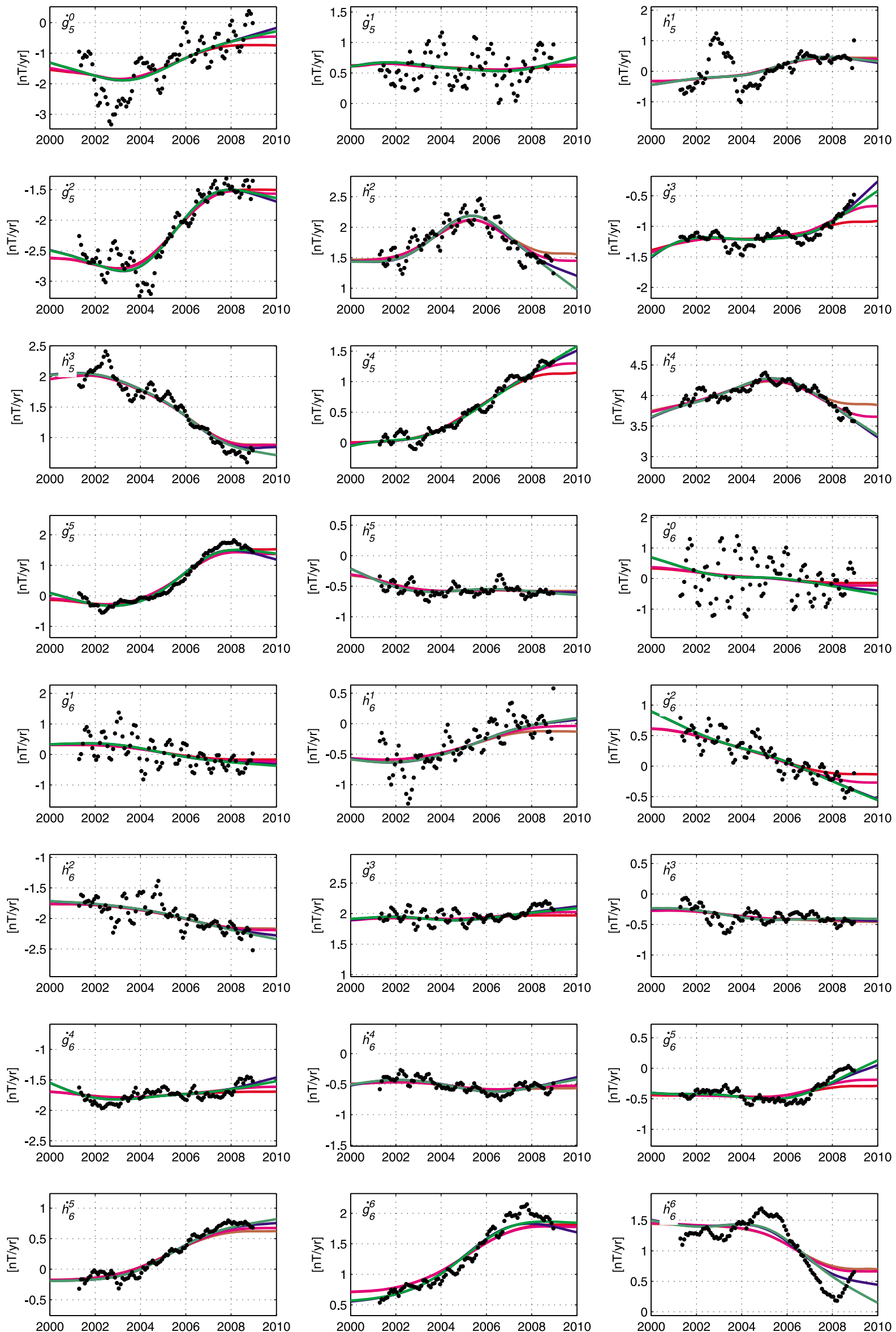

Fig. 4. Continuation of Fig. 3. 

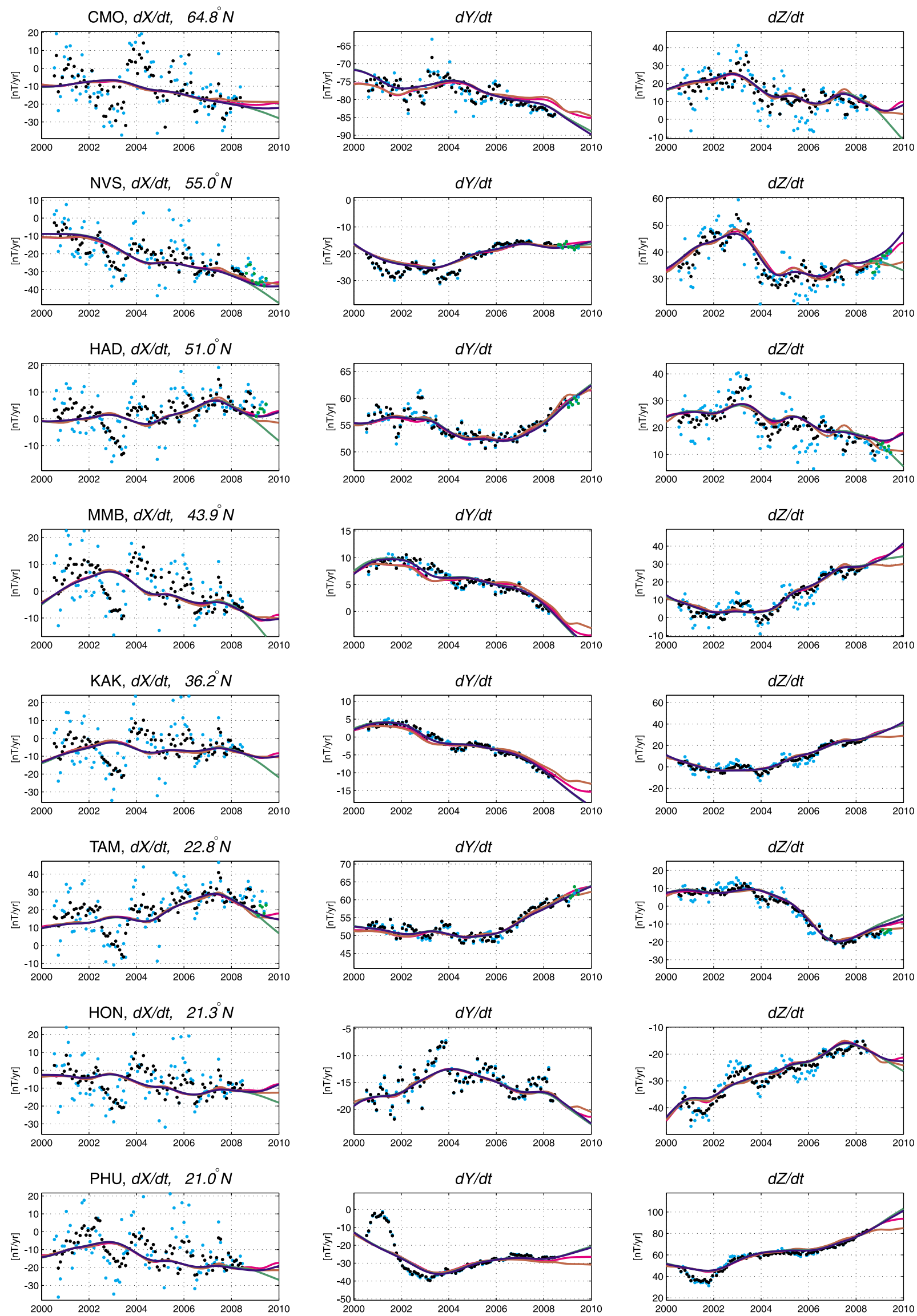

Fig. 5. First time derivative of the vector components, $d X / d t, d Y / d t$ and $d Z / d t$, at selected observatories. Symbols refer to observations (annual difference of revised monthly means in black, annual differences of traditional monthly means in light blue), whereas the solid curves indicate field estimates from the CHAOS-2s (red), "extended CHAOS-2s" (magenta), CHAOS-3 $\alpha$ (green) and CHAOS-3 (blue) models. Green symbols indicate values used for CHAOS-3 (and the updated CHAOS-2s model), but not for CHAOS-3 $\alpha$. 

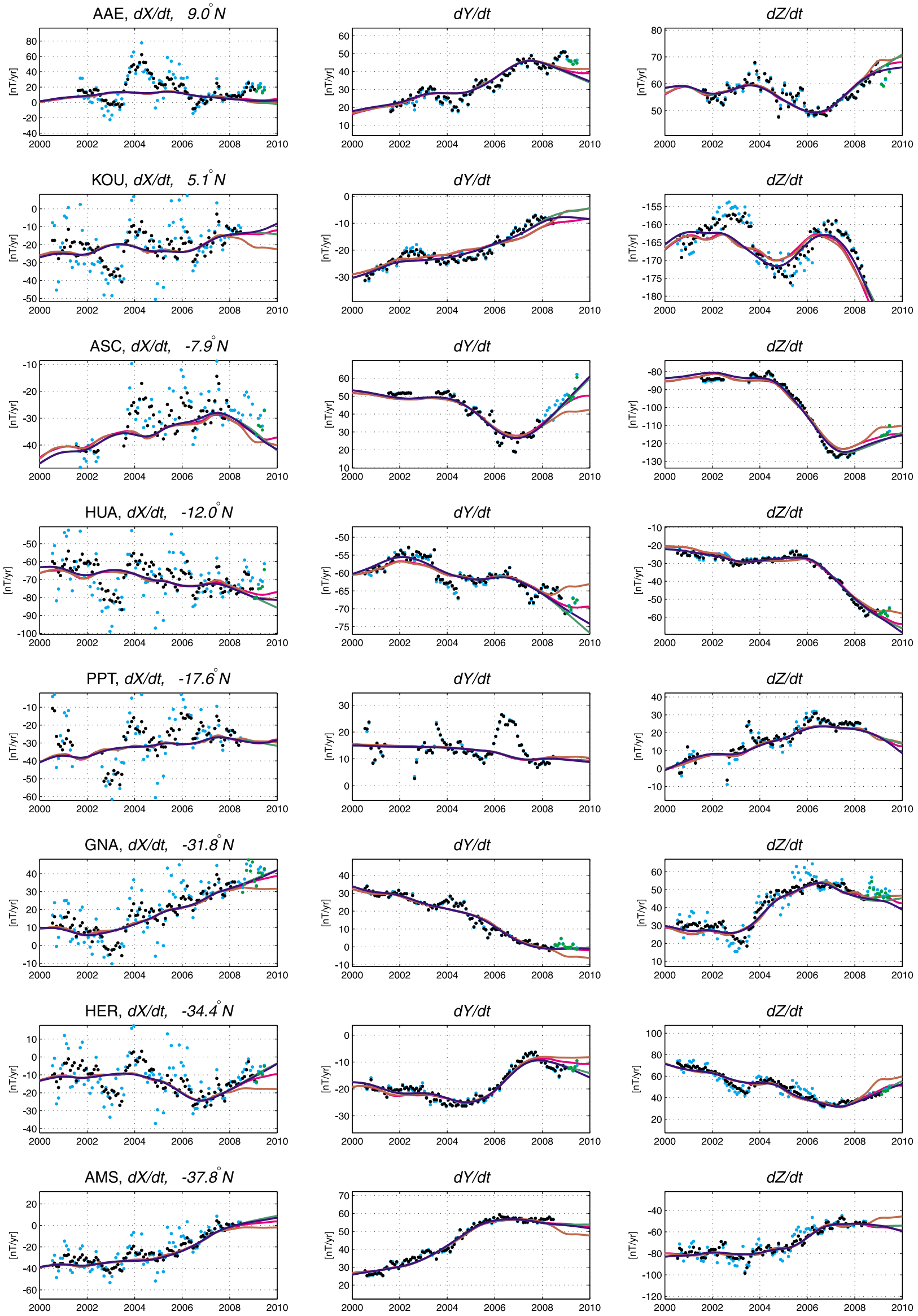

Fig. 6. Continuation of Fig. 5. 
ally high geomagnetic activity) at the observatories MMB, KAK, PHU, KOU, HUA and HER is an indication of this.

From the parent model CHAOS- $3 \alpha$ our three candidate models for IGRF-11 have been derived in the following way:

- our candidate model for DGRF-2005 is the degree $n=$ 1-13 part of CHAOS-3 $\alpha$ computed for the epoch $t=$ 2005.0;

- our candidate model for IGRF-2010 is the degree $n=1-13$ part of CHAOS- $3 \alpha$ computed for the epoch $t=2010.0$ (note that 2010 is the last spline knot of this model, but since only data until August 2009 have been used to determine the parent model CHAOS- $3 \alpha$, an extrapolation in time beyond the data span is performed);

- our candidate model for an average secular variation from 2010.0 to 2015.0 is the degree $n=1-8$ part of the first time derivative of CHAOS- $3 \alpha$ computed for the epoch $t=2010.0$.

Using satellite and observatory data (selected and processed as indicated before), high quality models of the recent geomagnetic field have been developed. They provide a detailed picture of the internal field, of the core field secular variation and secular acceleration at Earth's surface. Moreover, over the last decade, these models have dramatically improved the core field description at the core-mantle boundary and brought new insights into core dynamics.

Coefficients and data sets for the CHAOS- $3 \alpha$ and CHAOS-3 model versions are available at www.space.dtu.dk/files/magnetic-models/CHAOS-3/.

Acknowledgments. The Ørsted Project was made possible by extensive support from the Danish Government, NASA, ESA, CNES, DARA and the Thomas B. Thriges Foundation. The support of the CHAMP mission by the German Aerospace Center (DLR) and the Federal Ministry of Education and Research is gratefully acknowledged. We would like to thank the staff of the geomagnetic observatories and INTERMAGNET for supplying high-quality observatory data, and Susan MacMillan for providing us with preliminary observatory hourly mean values for 2009 . The work by MM is considered as IPGP contribution 3071.

\section{References}

Chulliat, A. and K. Telali, World monthly means database project, Publs. Inst. Geophys. Pol. Acad. Sc., C-99(398), 268-274, 2007.

Finlay, C. C., S. Maus, C. D. Beggan, M. Hamoudi, F. J. Lowes, N. Olsen, and E. Thébault, Evaluation of candidate geomagnetic field models for IGRF-11, Earth Planets Space, 62, this issue, 787-804, 2010.

Mandea, M. and N. Olsen, A new approach to directly determine the secular variation from magnetic satellite observations, Geophys. Res. Lett., 33, L15,306, doi:10.1029/2006GL026616, 2006.

Maus, S. and P. Weidelt, Separating the magnetospheric disturbance magnetic field into external and transient internal contributions using a 1D conductivity model of the Earth, Geophys. Res. Lett., 31, L12,614, doi:10.1029/2004GL020232, 2004.

Olsen, N., External field contributions in observatory monthly means, Geophys. Res. Abstracts, 11, 2009.

Olsen, N. and M. Mandea, Investigation of a secular variation impulse using satellite data: The 2003 geomagnetic jerk, Earth Planet. Sci. Lett., 255, 94-105, doi:10.1016/j.epsl.2006.12.008, 2007.

Olsen, N. and M. Mandea, Rapidly changing flows in the Earth's core, Nature Geosci., 1(6), 390, 2008.

Olsen, N., T. J. Sabaka, and F. Lowes, New parameterization of external and induced fields in geomagnetic field modeling, and a candidate model for IGRF 2005, Earth Planets Space, 57, 1141-1149, 2005.

Olsen, N., H. Lühr, T. J. Sabaka, M. Mandea, M. Rother, L. TøffnerClausen, and S. Choi, CHAOS-a model of Earth's magnetic field derived from CHAMP, Ørsted, and SAC-C magnetic satellite data, Geophys. J. Int., 166, 67-75, doi:10.1111/j.1365-246X.2006.02959.x, 2006.

Olsen, N., M. Mandea, T. J. Sabaka, and L. Tøffner-Clausen, CHAOS2-A geomagnetic field model derived from one decade of continuous satellite data, Geophys. J. Int., 179(3), 1477-1487, doi:10.1111/j.1365246X.2009.04386.x, 2009.

Sabaka, T. J., N. Olsen, and M. E. Purucker, Extending comprehensive models of the Earth's magnetic field with Ørsted and CHAMP data, Geophys. J. Int., 159, 521-547, doi:10.1111/j.1365246X.2004.02421.x, 2004.

N. Olsen (e-mail: nio@space.dtu.dk), M. Mandea, T. J. Sabaka, and L. Tøffner-Clausen 